\title{
Safety and Efficacy of the StarClose Vascular Closure System following 8-Fr Sheath Placement for Intra-Aortic Balloon Pump: A Single-Center Analysis of 42 Consecutive Patients
}

\author{
Guang Chu Wenyi Yang Guobing Zhang Zhi Zhang Shaowen Liu \\ Baogui Sun Fang Wang \\ Department of Cardiology, Shanghai Jiaotong University, First People's Hospital, Shanghai, PR China
}

\section{Key Words}

Intra-aortic balloon pump - Vascular closure device .

StarClose $\cdot$ Femoral artery

\begin{abstract}
Objective: To assess the safety and efficacy of the StarClose device following intra-aortic balloon pump (IABP) counterpulsation using 8-Fr femoral sheaths. Subjects and Methods: From June 2008 to August 2012, 42 consecutive patients who received IABP implantation via common femoral artery (CFA) punctures with an 8-Fr sheath (which were then sealed with the StarClose Vascular Closure System at the bedside) were included in this retrospective single-arm study. All the patients underwent duplex control of the puncture site $24 \mathrm{~h}$ after deployment of the device, in order to determine the presence or absence of vascular complications including hematoma, pseudoaneurysm, arteriovenous fistula and arterial/venous thrombosis or stenosis. The safety end points were the vascular complications during the hospital stay, and the efficacy end points included device and procedure success. Results: The procedure success rate was $92.9 \%$ (39/42) and the device success rate was $88.1 \%$ (37/42). Major vascular complications occurred in $3(7.1 \%)$ patients; 1 developed a massive hematoma $>10 \mathrm{~cm}$ which was man-
\end{abstract}

aged by blood transfusion and surgical reconstruction, and the other 2 developed pseudoaneurysm which was cured by ultrasound-guided thrombin injection or manual compression. Minor vascular complications occurred in 5 (11.9\%) patients, including blood oozing in 2, hematoma $<5 \mathrm{~cm}$ in 2 and severe pain in the remaining patient. Conclusion: CFA closure with the StarClose device was safe, feasible and effective in patients undergoing IABP support using 8- $\mathrm{Fr}$ sheath sizes.

(c) 2014 S. Karger AG, Basel

\section{Introduction}

Vascular interventional procedures, especially intraaortic balloon pump (IABP) implantation, are still often performed through femoral access. Manual compression to the femoral artery is a successful method of hemostasis and is widely used. However, there are other arterial closure devices which have been reported to allow quicker and more convenient hemostasis and increase patient comfort as well as facilitating rapid ambulation [1-4]. The StarClose Vascular Closure System has some practical advantages over the other devices, as it closes the arteriotomy without an intraluminal component. This de-

\begin{tabular}{ll}
\hline KARGER & $\begin{array}{l}\text { @ 2014 S. Karger AG, Basel } \\
1011-7571 / 14 / 0234-0313 \$ 39.50 / 0 \quad \text { Karger }\end{array}$ \\
E-Mail karger@karger.com & $\begin{array}{l}\text { This is an Open Access article licensed under the terms of the } \\
\text { Creative Commons Attribution-NonCommercial 3.0 Un- } \\
\text { ported license (CC BY-NC) (www.karger.com/OA-license), } \\
\text { applicable to the online version of the article only. Distribu- } \\
\text { tion permitted for non-commercial purposes only. }\end{array}$
\end{tabular}

Wenyi Yang, MD, or Fang Wang, PhD, MD

Department of Cardiology, Shanghai Jiaotong University

First People's Hospital, 85 Wu Jin Road

Shanghai 200080 (PR China)

E-Mail etaloc@163.com 
vice has been proven to not be inferior to manual compression with regard to major vascular events for the closure of 5-, 6- and 7-Fr sheaths [2, 5-11]. Although the StarClose for closure of the 8-Fr common femoral artery (CFA) sheath access sites following peripheral vascular interventions is far beyond the approved range of arteriotomies, it appears to be effective in maintaining hemostasis in a few studies $[2,3]$. However, there is no study reporting the use of StarClose in patients who have received IABP counterpulsation support with an 8-Fr sheath. In this study, we sought to assess the safety and efficacy of StarClose vascular closure of 8-Fr CFA sheath access in patients who undergo IABP implantation.

\section{Subjects and Methods}

\section{Subjects}

Between June 2008 and August 2012, 156 patients who were admitted to the Department of Cardiology with a diagnosis of acute myocardial infarction underwent percutaneous coronary intervention and IABP support (Arrow International Inc., Maynard, Mass., USA) using an 8-Fr femoral sheath. Manual compression was performed in 114 patients after removing the IABP. Patients with severe calcification, severe stenosis, dissection or distortion of the femoral or iliac arteries were excluded. The remaining 42 patients (28 males and 14 females with a mean age of $61.5 \pm 9.1$ years, range 48-75 years), who were treated with the StarClose Vascular Closure System (Abbott Vascular, Redwood City, Calif., USA) for hemostasis with the official permission from the Ethics Committee of the hospital, were enrolled in this retrospective, single-arm, single-center study.

\section{Methods}

Forty-two consecutive patients were given $600 \mathrm{mg}$ of clopidogrel and $300 \mathrm{mg}$ of aspirin as a loading dose at least $2 \mathrm{~h}$ before the coronary intervention and IABP implantation and then 5,00010,000 units of heparin intravenously during the procedure. After the procedure, all subjects continued to receive dual antiplatelet therapy with aspirin $(100 \mathrm{mg} /$ day $)$ and clopidogrel $(75 \mathrm{mg} /$ day $)$ and enoxaparin hypodermically at a dose of 80-160 mg/day for 1 week. Tirofiban hydrochloride was administered at a dose of $0.3-$ $0.5 \mathrm{mg} / \mathrm{h}$ for $36-48 \mathrm{~h}$ in 8 patients. IABP support lasted for $2-7$ days (table 1).

All patients were treated at the bedside by 3 operators (G.C., W.Y. and G.Z.) trained in the use of the StarClose device. First, the IABP-dedicated 8-Fr sheath was replaced with a StarClose-dedicated sheath with a guide wire $(0.025$ inches $)$ that was placed into the ascending aorta via the IABP pressure chamber. Second, the 4-mm, flexible, star-shaped nitinol clip of the StarClose was employed to complete a circumferential, extravascular, puncture site closure. Finally, a sterile wound dressing with 5-min manual compression was applied to the puncture site, and the patient was required to remain in bed for $4 \mathrm{~h}$. In case of device failure, an additional pressure bandage was applied, followed by $6 \mathrm{~h}$ of immobilization. A duplex scan of the groin was performed the day after the
Table 1. Baseline characteristics and procedure details in the 42 patients

\begin{tabular}{lc}
\hline Age, years & $61.5 \pm 9.1$ \\
Men & $28(66.7)$ \\
STEMI & $26(61.9)$ \\
NSTEMI & $16(38.1)$ \\
\hline Risk factors & \\
History of tobacco use & $25(59.5)$ \\
Diabetes mellitus & $18(42.9)$ \\
Hyperlipoproteinemia & $15(35.7)$ \\
BMI & $22.8 \pm 3.6$ \\
Obesity & $20(47.6)$ \\
Hypertension & $23(54.8)$ \\
LVEF, \% & $42 \pm 5.7$ \\
Scr, $\mu$ mol/l & $97 \pm 8.6$ \\
\hline Procedure details & \\
Heparin, units & $6,597.3 \pm 959.2$ \\
Enoxaparin, mg/day & $115.5 \pm 11.5$ \\
$\quad$ Time, days & $4.2 \pm 1.8$ \\
Glycoprotein IIb/IIIa inhibitor & \\
$\quad$ (tirofiban) & $8(19.0)$ \\
Time, h & $40.5 \pm 5.6$ \\
IABR support, days & $1.05 \pm 0.21$ \\
& $3.5 \pm 1.2$
\end{tabular}

Continuous data are presented as mean \pm standard deviation; categorical data are given as $\mathrm{n}(\%)$.

$\mathrm{BMI}=$ Body mass index; INR = international normalized ratio; LVEF = left ventricular ejection fraction; NSTEMI = non-ST-segment elevation myocardial infarction; Scr = serum creatinine; STEMI = ST-segment elevation myocardial infarction.

procedure to examine the external iliac artery, CFA, superficial and deep femoral arteries, and corresponding veins, in order to assess the effectiveness of closure and the presence of any complication.

The primary safety end point was the composite incidence of major vascular injuries requiring surgical or interventional repair (e.g. acute access site bleeding, hematoma, pseudoaneurysm, arteriovenous fistula, arterial/venous thrombosis or stenosis and new ipsilateral lower extremity ischemia), access site bleeding requiring transfusion, access site nerve injury requiring intervention and access site infection requiring intravenous antibiotics or a prolonged hospital stay. The secondary safety end point was the composite incidence of minor vascular injuries including arteriovenous fistula, hematoma, late access site bleeding (oozing) without the need for further intervention, transient lower extremity ischemia and ipsilateral deep venous thrombosis (DVT).

The primary efficacy end point was device success, which was defined as successful hemostasis by using the StarClose device alone or after $<5$ min of adjunctive compression and freedom from major vascular complications, as listed above. The secondary efficacy end point was the procedure success at discharge, which was defined as the attainment of final hemostasis by using any method and freedom from major vascular complications. 


\section{Results}

The baseline demographic data and clinical characteristics of the patients are presented in table 1.

\section{Device Safety}

There was no procedure-related death throughout the study. Major vascular complications occurred in 3 (7.1\%) of the 42 patients, including a nonobese male patient who received glycoprotein IIb/IIIa inhibitors for $48 \mathrm{~h}$ and IABP support for 3 days and developed access site bleeding and a massive hematoma $>10 \mathrm{~cm}$ that required transfusion and surgical reconstruction after device deployment. Pseudoaneurysm occurred in $2(4.8 \%)$ patients, including a nonobese woman who received IABP support for 4 days without using glycoprotein IIb/IIIa inhibitors, where the complication was treated successfully with manual compression. The other patient was an obese man who received glycoprotein IIb/IIIa inhibitors for $36 \mathrm{~h}$ and IABP support for 5 days, where the complication was treated successfully with ultrasound-guided thrombin injection. There was no nerve injury that required intervention, no new ipsilateral acute lower limb ischemia caused by severe stenosis or dissection and no infection that required intravenous antibiotic therapy (table 2).

Minor vascular complications occurred in 5 (11.9\%) patients, including blood oozing in 2, which was cured after manual compression for 20 and $40 \mathrm{~min}$, respectively, hematoma $<5 \mathrm{~cm}$ in 2 and severe pain in the remaining patient (which was managed by applying topical analgesics to the puncture site during the first $6 \mathrm{~h}$ after the procedure). No arteriovenous fistulas or ipsilateral DVT occurred in any patients (table 2).

\section{Device Efficacy}

Device failure occurred in 3 cases and major complications in 3 cases (including 1 of the cases of device failure), so the overall device success rate was $88.1 \%(37 / 42)$. Of the 3 device failures (in 2 men and 1 woman), 2 patients were hypertensive, 2 were diabetic, 1 was overweight and 1 received glycoprotein IIb/IIIa inhibitors (IABP support lasted for 3-6 days for this patient). Two patients were treated successfully with conventional manual compression. One patient developed a pseudoaneurysm that was resolved after ultrasound-guided compression. Therefore, the procedure success rate (including hemostasis by any means without occurrence of major complications) in the 42 patients was $92.9 \%(39 / 42)$.

The Use of StarClose in Patients with IABP

Table 2. Device efficacy and vascular complications in the $42 \mathrm{pa}$ tients

$\begin{array}{ll}\text { Device success } & 37(88.1) \\ \text { Procedure success } & 39(92.9) \\ \text { Death } & 0(0) \\ \text { Major vascular complications } & 3(7.1) \\ \quad \text { Vascular injury requiring repair } & \\ \quad \text { Surgery } & 1(2.4) \\ \quad \text { Angioplasty } & 0(0) \\ \quad \text { Ultrasound-guided compression } & 1(2.4) \\ \quad \text { Thrombin injection } & 1(2.4) \\ \quad \text { Other percutaneous procedures } & 0(0) \\ \text { New ipsilateral lower extremity ischemia } & 0(0) \\ \text { Access site bleeding requiring transfusion } & 1(2.4) \\ \text { Access site infection requiring IV antibiotics } & 0(0) \\ \text { Access site nerve injury } & 0(0) \\ \text { Minor vascular complications } & 5(11.9) \\ \text { Arteriovenous fistula } & 0(0) \\ \text { Hematoma } & 2(4.8) \\ \text { Oozing } & 2(4.8) \\ \text { Transient lower extremity ischemia } & 0(0) \\ \text { Intense, persistent (>6 h) pain } & \\ \text { at the puncture site } & 1(2.4) \\ \text { Ipsilateral DVT } & 0(0)\end{array}$

Values are presented as n (\%). IV = Intravenous.

\section{Discussion}

We achieved a device success rate of $88.1 \%$ and a procedure success rate of $92.9 \%$ for hemostasis using the StarClose device. In addition, there were very few complications, and all of these were amenable to treatment with surgical and endovascular methods. The $7.1 \%$ major vascular complications and $11.9 \%$ minor complications attending the use of the StarClose device are similar to the $15 \%$ vascular complications (11\% were major) reported for conventional manual compression $[12,13]$. Hence, it can be deduced that the StarClose device was not inferior to standard manual compression in terms of safety and efficacy. Branzan et al. [2] and Das et al. [3] demonstrated that CFA closure with the StarClose device following coronary or peripheral vascular interventions utilizing $8-\mathrm{Fr}$ sheath sizes was feasible and had few device-specific complications. Similar results were obtained in this study, which further confirmed the safety and efficacy of the StarClose device in patients with introducer sheaths $(>6$ Fr) during the catheterization procedure. However, our study differed from Branzan et al. [2] and Das et al. [3]. The StarClose device was used in patients who received 
IABP support, which had not yet been systematically examined. Moreover, the closure procedure was developed not at the cardiac catheterization laboratory but at the bedside, and not immediately but $2-7$ days after coronary intervention. Although the StarClose device for the closure of 8-Fr CFA sheath access sites following vascular interventions is far beyond the approved range of arteriotomies, it theoretically allows the use of a sheath larger than the size of the clip-based closure device, since the diameter of the clip is $>8 \mathrm{Fr}$. At the same time, a device that closes the arteriotomy without an intraluminal component, reducing the risk of local thrombosis and lumen narrowing, could represent an advance in arterial closure, and may have some practical advantages over the AngioSeal or Perclose ProGlide devices that have been licensed for 8-Fr sheath use [14-17].

The $88.1 \%$ device success rate of this study was within the range of the $87-94 \%$ device success rate $[3,9,18-21]$, but the $92.9 \%$ was slightly lower than the $96-100 \%$ procedural success rate $[2,3,19-23]$ reported for the same device in 5-, 6- or 7-Fr punctures. The 7.1\% major vascular complication rate attributable to StarClose in this study was higher than previously reported $0-4.1 \%[2-6$, $9,18-24]$, but the minor $11.9 \%$ complication rate was within the range of $2.1-27 \%$, even though the device was used for closing larger puncture holes.

The discrepancies between studies could be due to the following reasons. The duplex scan was performed the day after the procedure as a routine for the 42 patients, rather than when hematoma or bruit was detected on physical examination. As a result, clinically asymptomatic vascular complications were detected earlier, thus increasing the diagnostic rate compared to some other studies $[3,6,20,22,24]$ in which the duplex scan was not used routinely. All patients in this study received dual antiplatelet therapy with aspirin and clopidogrel and anticoagulant therapy. Glycoprotein IIb/IIIa inhibitors were utilized in $19.0 \%$ of patients. However, the patients undergoing diagnostic catheterization only took aspirin $[7,8,24]$. Anticoagulation treatment was not routinely used after peripheral angioplasty procedures $[6,19,20]$. In the study by Branzan et al. [2], 14.8\% of the patients received glycoprotein IIb/IIIa inhibitor therapy. As the antithrombotic therapy in our series was significantly stronger than that of these studies, the occurrence of DVT was decreased at the expense of an increased risk of oozing, hematoma and pseudoaneurysm. No new ipsilateral acute lower limb ischemia caused by vascular injury occurred after using the StarClose device. However, device misplacement with subsequent vascular stenosis and the need for surgical removal and vascular reconstruction has already been reported [25]. An explanation might be that femoral angiography was performed to exclude severe stenosis, severe calcification, dissection, distortion or vascular malformations before IABP support procedures. In other studies [2, 4-7, 18-20, 22-24], the StarClose device was used for hemostasis immediately after diagnostic and interventional catheterizations whereas in our study, the procedure was performed 2-7 days after IABP insertion. The elasticity of 8-Fr holes was poor, and the blood exuded easily through the puncture channel after deployment of the StarClose, which might be the reason for the higher rate of bleeding complications in our series. The StarClose devices were all used at the bedside, rather than under fluoroscopy in the catheter laboratories as in most other studies, which might have increased the device success rate. However, it was not safe enough to transport the patients with IABP to the laboratory. Moreover, the use of StarClose at the bedside would, theoretically, increase the chance of infection, but there was no access site infection requiring intravenous antibiotics. The reason could be that we expanded the scope of disinfection effectively and followed the principles of aseptic manipulation strictly. In this study, additional 5-min manual compression was routinely performed after deployments according to the instruction. Longer manual compression would be helpful to decrease the complications in the case of 2-7 days of delayed deployment, an 8-Fr sheath and stronger antithrombotic strategies.

The main limitation of this study was its retrospective nature and inherent selection bias. The sample size in the single-center series was relatively small. In addition, as the indication for manual compression or StarClose deployment was not standardized, the decision was left to the discretion of the operator.

\section{Conclusion}

This study provided additional information on the clinical use of the clip-based StarClose sealing device, and demonstrated that this device is effective for hemostasis after IABP procedures using 8-Fr femoral sheaths. However, randomized controlled trials are needed to be able to compare manual compression and the StarClose vascular closure device in these clinical settings. 


\section{References}

$>1$ Madigan JB, Ratnam LA, Belli AM: Arterial closure devices. A review. J Cardiovasc Surg 2007;48:607-624.

2 Branzan D, Sixt S, Rastan A, et al: Safety and efficacy of the StarClose Vascular Closure System using 7-F and 8-F sheath sizes: a consecutive single-center analysis. J Endovasc Ther 2009; 16:475-482.

$\checkmark 3$ Das R, Ahmed K, Athanasiou T, et al: Arterial closure devices versus manual compression for femoral haemostasis in interventional radiological procedures: a systematic review and meta-analysis. Cardiovasc Intervent Radiol 2011;34:723-738.

4 Tavris DR, Wang Y, Jacobs S, et al: Bleeding and vascular complications at the femoral access site following percutaneous coronary intervention (PCI): an evaluation of hemostasis strategies. J Invasive Cardiol 2012;24:328334.

$>5$ Jaff MR, Hadley G, Hermiller JB, et al: The safety and efficacy of the StarClose Vascular Closure System: the ultrasound substudy of the CLIP study. Catheter Cardiovasc Interv 2006;68:684-689.

6 Spiliopoulos S, Katsanos K, Karnabatidis D, et al: Safety and efficacy of the StarClose vascular closure device in more than 1,000 consecutive peripheral angioplasty procedures. J Endovasc Ther 2011;18:435-443.

$\checkmark 7$ Chiu AH, Coles SR, Tibballs J, et al: The StarClose vascular closure device in antegrade and retrograde punctures: a single-center experience. J Endovasc Ther 2010;17:46-50.

$>8$ Tapping CR, Dixon S, Little MW, et al: StarClose arterial closure after conversion from retrograde to antegrade access. Clin Radiol 2012;67:889-892.

$\checkmark 9$ Rodriguez A, Katz SG: The use of the StarClose device for obtaining femoral artery hemostasis. Vasc Endovascular Surg 2011;45: 627-630.
10 Burke MN, Hermiller J, Jaff MR: StarClose Vascular Closure System (VCS) is safe and effective in patients who ambulate early following successful femoral artery access closure results from the RISE clinical trial. Catheter Cardiovasc Interv 2012;80:45-52.

11 Radak D, Babic S, Peric M, et al: Distribution of risk factors in patients with premature coronary, supra-aortic branches and peripheral atherosclerotic disease. Med Princ Pract 2012; 21:228-233.

12 Cohen M, Dawson MS, Kopistansky C, et al: Sex and other predictors of intra-aortic balloon counterpulsation-related complications: prospective study of 1,119 consecutive patients. Am Heart J 2000;139:282-287.

13 Chadow HL, Hauptman RE, Strizik B, et al: Vasoseal after intra-aortic balloon pump removal: a pilot study. Catheter Cardiovasc Interv 2000;50:495-497.

14 Wille J, Vos J, Overtoom TT, et al: Acute leg ischemia: the dark side of a percutaneous femoral artery closure device. Ann Vasc Surg 2006;20:278-281.

15 Kadner A, Schmidli J, Schwegler I, et al: Complications associated with the arterial puncture closure device - Angio-Seal. Vasc Endovascular Surg 2008;42:225-227.

16 Van Den Broek T, Liqui Lung PF, Suttorp MJ, et al: Vascular occlusion as a late complication of the Angio-Seal closure device. A review of literature. Minerva Cardioangiol 2007;55: 815-819.

17 Mackrell PJ, Kalbaugh CA, Langan EM 3rd, et al: Can the Perclose suture-mediated closure system be used safely in patients undergoing diagnostic and therapeutic angiography to treat chronic lower extremity ischemia? J Vasc Surg 2003;38:1305-1308.
18 Williams RE, Angel CY, Bourkaib R, et al: Multicenter safety and efficacy analysis of assisted closure after antegrade arterial punctures using the StarClose device. J Endovasc Ther 2007;14:498-505.

19 Imam A, Carter RM, Phillips-Hughes J: StarClose vascular closure device: prospective study on 222 deployments in an interventional radiology practice. Cardiovasc Intervent Radiol 2007;30:738-742.

20 Tay EL, Co M, Tai BC, et al: Clinical experience of StarClose vascular closure device in patients with first and recurrent femoral punctures. J Interv Cardiol 2008;21:67-73.

21 Rastan A, Sixt S, Frank U, et al: VIPER-2: a prospective, randomized single-center comparison of 2 different closure devices with a hemostatic wound dressing for closure of femoral artery access sites. J Endovasc Ther 2008;15:83-90.

22 Ratnam LA, Raja J, Munneke GJ, et al: Prospective nonrandomized trial of manual compression and Angio-Seal and StarClose arterial closure devices in common femoral punctures. Cardiovasc Intervent Radiol 2007;30: 182-188.

23 Desideri A, Tonello D, Coscarelli S, et al: Early mobilization after percutaneous catheterization and vascular closure with a novel device (StarClose). Am J Cardiol 2005;96:14081409.

24 Hermiller J, Simonton C, Hinohara T, et al: Clinical experience with a circumferential clip-based vascular closure device in diagnostic catheterization. J Invasive Cardiol 2005;17: 504-510.

-25 Stock U, Flach P, Gross M, et al: Intravascular misplacement of an extravascular closure system: StarClose. J Interv Cardiol 2006;19:170172 